\title{
Factors related to the treatment duration of infants with congenital muscular torticollis
}

\author{
Seonghyeok Song $\left.{ }^{\mathrm{a}(}\right)$, Wonjeong Hwang ${ }^{\mathrm{b}(\infty)}$, Seungwon Lee ${ }^{\mathrm{c}(1)}$ \\ ${ }^{a}$ Department of Pediatric Physical Therapy, Ez Rehabilitation Medical Center, Yongin, Republic of Korea \\ ${ }^{\mathrm{b}}$ Research Facility of Academy of Women's Health in Physical Therapy, Sahmyook University, Seoul, Republic of Korea \\ ${ }^{\mathrm{c} D e p a r t m e n t ~ o f ~ P h y s i c a l ~ T h e r a p y, ~ C o l l e g e ~ o f ~ H e a l t h ~ S c i e n c e ~ a n d ~ S o c i a l ~ W e l f a r e, ~ S a h m y o o k ~ U n i v e r s i t y, ~ S e o u l, ~ R e p u b l i c ~ o f ~ K o r e a ~}$
}

Objective: Congenital muscular torticollis (CMT) is a disease with abnormal head and neck posture in infants. It affects the child's movement and development and can lead to complications. Therefore, this study aims to find out what factors influence the rehabilitation treatment duration of infants with CMT.

Design: Cross-sectional study.

Methods: The subjects were 63 infants under 90 days of age who were diagnosed with CMT. Age, thickness of the sternocleidomastoid muscle (SCM) on the affected and non-affected side, head tilt angle, and head rotation angle of the affected head in infants were collected. The ratio of muscle thickness was calculated from the thickness of the affected SCM and the thickness of the non-affected SCM (A/N ratio). All subjects underwent conservative physical therapy twice a week for 30 minutes, and the end of the treatment was when the angle of head tilt was normal or less than 5 degrees, and the treatment duration was calculated.

Results: Age, thickness of affected SCM, and head tilt were significantly correlated with treatment duration $(p<0.05)$. The thickness of the non-affected SCM, A/N ratio, and head rotation angle did not show any correlation with treatment duration. The factors affecting the treatment duration were head tilt and age, showing $21 \%$ explanatory power of adjusted $\mathrm{R}^{2}$.

Conclusions: The main factors affecting the treatment duration of infants with CMT are head tilt and age. Therefore, more attention should be directed to the infant's head tilt and age for effective physical therapy of infants with CMT.

Key Words: Congenital torticollis, Prognosis, Rehabilitation

\section{Introduction}

In congenital muscular torticollis (CMT), the thickness of the sternocleidomastoid muscle (SCM) on one side increases and the length is shortened, which leads to cervical flexion to the same side and rotation to the opposite side $[1,2]$.

CMT is classified into three categories: a sternocleidomatoid tumor group in which a tumor is observed in the pectoralis muscle, muscular torticollis in which only muscle tension is observed, and a postural torticollis in which no mass or tension is felt. The causes of congenital muscular torsion includes malposition, trauma during delivery, and compart- ment syndrome, but the cause of congenital muscular torsion is unknown [2-5]. CMT causes hip dysplasia, plagiocephaly, and cervical scoliosis, and has a negative body image, which negatively affects the quality of life.

The general treatment method is to perform passive stretching on the shortened SCM $[3,5,6]$.

It was reported that $90 \%$ of patients with CMT were able to recover through stretch exercise and only $10 \%$ needed surgical therapy [7]. However, there are many disagreements regarding the application of surgery to children under the age of 1 [8].

To increase cervical range of motion, passive and active extensions are performed [3]. However, since stretch ex-

Received: 31 August, 2020 Revised: 15 September, 2020 Accepted: 17 September, 2020

Corresponding author: Seungwon Lee (ORCID https://orcid.org/0000-0002-0413-0510)

Department of Physical Therapy, College of Health and Welfare, Sahmyook University, 815 Hwarang-ro, Nowon-gu, Seoul 01795, Republic of Korea Tel: 82-2-3399-1630 Fax: 82-2-3399-1639 E-mail: swlee@syu.ac.kr

(c) This is an Open-Access article distributed under the terms of the Creative Commons Attribution Non-Commercial License (http://creativecommons.org/licenses/ by-nc/4.0) which permits unrestricted non-commercial use, distribution, and reproduction in any medium, provided the original work is properly cited.

Copyright @ 2020 Korean Academy of Physical Therapy Rehabilitation Science 
ercise has some disadvantage of pain or resistance, active movement is emphasazied, and recently, treatement is approached in a variety of ways, including the application of Tscharnuter Akademie for Motor Organization therapy, which induces active participation of patients, [9] as well as soft tissue mobilization [10]. Early diagnosis and treatment of torticollis is important. Patients diagnosed with CMT more than 1 month after birth had a longer treatment duration than those diagnosed in less than 1 month [3]. As a result of comparing the recovery rates of patients diagnosed before and after 6 weeks of age, more optimal recovery rates were found in the patients diagnosed before 6 weeks of age [11]. Good prognosis has been demonstrated with performing physical therapy within 3 months, and it has been reported that the greater the time of delay in receiving physical therapy, the greater the number of cases requiring surgical methods $[12,13]$. If there is no response to treatment until the age of 1 , surgery should be considered [11]. Particularly after 3 months of age, actions to adjust the neck and move voluntarily appeared, but when trying to turn the head toward the affected side, the entire body must be turned, one shoulder is raised, and it is difficult to level the eyes. Because the head cannot be placed in the center, midline orientation is difficult to establish, and so the field of view is skewed to one side, and the opportunity for two-hand coordination is lost, such as the use of the hand on the side where the field of view is secured, making it difficult to achieve balanced left-right development. As such, early detection of CMT and early treatment can influence the development of the patient.

CMT is a widely known cervical spine disease where the importance of early treatment is also known, and if recovery is delayed, it is a disease that may lead to developmental problems and require future surgery. Therefore, it is important to clarify what factors affect the outcome of rehabilitation of children with CMT, but many studies on them are insufficient.

Therefore, the purpose of this study was to first investigate the factors related to the treatment duration of infants with CMT who are under 3 months of age. Second, the main factors affecting the treatment duration also warranted investigation.

\section{Methods}

\section{Participants}

For this study, data was collected from 63 children under 3 months of age out of 100 children who visited the E-re- habilitation department at the E-rehabilitation medicine department until treatment was terminated. Diagnosis of torticollis was made with a child who received the disease code [M4362], and subjects with neurological disorders such as ocular torticollis, congenital cervical spine problems, eye problems and upper respiratory tract infection were excluded. This study was approved by the Institutional Review Board of Sahmyook University (IRB No. 2-700179 3-AB-N-012019103HR).

\section{Procedures and Instruments}

Information about the patient's age (days), weight at birth $(\mathrm{kg})$, delivery type, and the mother's age at delivery before treatment were obtained through medical records. The age of the patient was the same as the day the diagnosis was received and the start of treatment.

A physical therapist with more than 7 years of clinical experience assessed the direction of the torticollis and the angles of head tilt and head rotation. The rehabilitation medicine doctor confirmed the hip joint dislocation through $\mathrm{X}$-ray and measured the thickness of the SCM.

The muscle thickness of the SCM was measured using a diagnostic ultrasonography (E-CUBE 11; Alpinion Medical Systems Co., Ltd., Anyang, Korea). With the patient lying in supine, the neck was slightly extended by placing a small pillow under the shoulder and slightly rotated to the other side.The SCM thickness of the affected side and the SCM thickness on the non-affected, healthy side were measured respectively, and the thickest part was measured while measuring along the muscle (Figure 1) $[14,15]$. The ratio between the SCM thickness on the affected side and the non-affected, healthy side was defined as the $\mathrm{A} / \mathrm{N}$ ratio

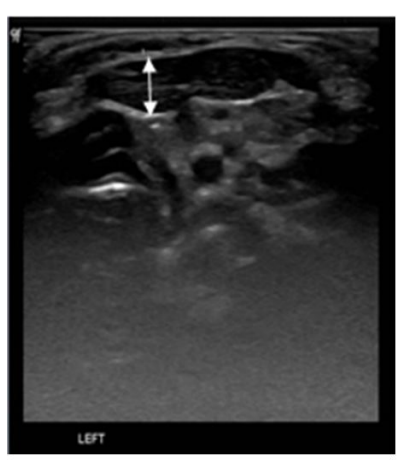

Left side

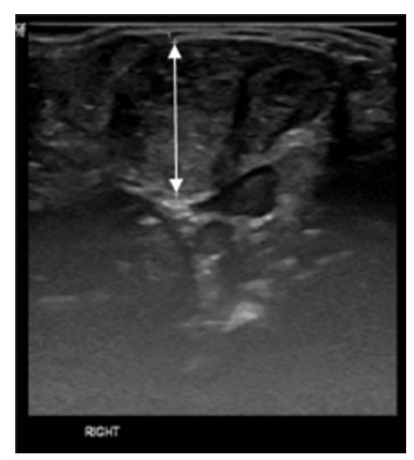

Right side
Figure 1. Ultrasound image of the thickness of the congenital muscular torticollis of a patient with the right lesion with longitudinal view. 


\section{$[14,15]$}

The head tilt angles and head rotation were measured using an arthrodial protractor (Vector diagnostics; Lafayette Medical Inc., Morgan Hill, CA, USA). The patient was laid in supine and the assistant fixed both shoulders of the patient, and the head tilt and head rotation angles were measured by passive range of motion (PROM) using an arthrodial protractor $[14,16]$. Each subject was subjected to physical therapy twice a week, 30 minutes each, by a physical therapist with more than 7 years of clinical experience in treating torticollis. Conservative treatment was administered followed by passive elongation, postural control and thermal therapy. For passive stretches, the patient was placed in a supine position and a passive extension intervention was performed to elongate the shortened SCM, which was maintained for 10 seconds at the end range. Depending on the patient's condition, the stretches were conducted 10-20 times with 1-minute rest period provided in, totaling a treatment time of 15 minutes.

As an intervention for postural control, the SCM of the affected side was elongated using eye tracting, neck righting, and tonic neck reflex, and the SCM of the nonaffected side was handled for 15 minutes so that it can be strengthened by itself. Heat therapy was provided with 15 minutes of therapeutic ultrasound (frequency $3 \mathrm{MHZ}$, intensity: 0.8-1.0 $\mathrm{W} / \mathrm{cm}^{2}$, ceramic size $1 \mathrm{~cm}$ ).

After each conservative treatment, 15 minutes of exercise

Table 1. General characteristics of participants

$(\mathrm{N}=63)$

\begin{tabular}{lc}
\hline \multicolumn{1}{c}{ Variable } & Value \\
\hline Sex (male/female) & $29 / 34$ \\
Age (d) & $70.22(18.92)$ \\
Direction (Rt./Lt.) & $39 / 24$ \\
Birth weight (kg) & $3.69(0.30)$ \\
NSVD/Cesarean section & $30 / 33$ \\
Dislocation/Normal & $21 / 42$ \\
Mom's age (y) & $32.00(1.56)$ \\
Treatment duration $(\mathrm{d})$ & $100.62(20.23)$ \\
A-SCMT (mm) & $1.81(0.28)$ \\
N-SCMT (mm) & $0.30(0.09)$ \\
A/N ratio & $6.33(1.53)$ \\
Head tilt $\left({ }^{\circ}\right)$ & $18.97(2.55)$ \\
Head rotation $\left(^{\circ}\right)$ & $50.00(4.21)$ \\
\hline
\end{tabular}

Values are presented as number only or Mean (SD).

Rt.: right, Lt.: left, NSVD: normal spontaneous vaginal delivery, A-SCMT: affected sternocleidomastoid muscle thickness, N-SCMT: non-affected sternocleidomastoid muscle thickness, $\mathrm{A} / \mathrm{N}$ ratio: affected sternocleidomastoid muscle thickness/non-affected sternocleidomastoid muscle thickness. therapy was performed to adjust the child's posture and use the large muscles according to the child's age and developmental stage.

As for parental education, postural education such as moving posture, sleeping posture, play posture, and other postural education were conducted in the same manner, and periodic monitoring was conducted. The treatment end point was when the angle of head tilt was normal or less than 5 degrees [17].

\section{Analysis}

For general information of the subject, the mean, standard deviation and number were used through descriptive analysis. A student t-test was conducted to determine the relationship between the treatment duration (days), sex, affected side of the torticollis, delivery type, and hip dislocation. Pearson correlation analysis was used to find out the relationship between the SCM thickness, A/N ratio, head tilt, and head rotation of the affected and non-affected sides.

The stepwise multiple regression model was used to find out the variables that affect the treatment duration. All data analyses were carried out using SPSS for Windows, Version 25.0 (IBM Co., Armonk, NY, USA). All statistical significance levels $(\alpha)$ were 0.05 .

\section{Results}

The general characteristics of the subjects are shown in (Table 1). The SCM thickness on the affected side was

Table 2. Relationship between treatment duration and variables

\begin{tabular}{lrc}
\hline \multicolumn{1}{c}{ Variable } & Length of treatment & $\mathrm{t}$ \\
\hline Sex & & 0.597 \\
$\quad$ Male & $102.28(23.30)$ & \\
$\quad$ Female & $99.21(17.44)$ & \\
Torticollis affected side & & -0.794 \\
$\quad$ Rt. & $99.03(19.21)$ & \\
$\quad$ Lt. & $102.21(21.97)$ & \\
Delivery & & -1.506 \\
$\quad$ NSVD & $99.63(20.19)$ & \\
$\quad$ Cesarean section & $104.24(19.88)$ & \\
Hip dislocation & & \\
$\quad$ Normal & $100.43(18.32)$ & 0.105 \\
$\quad$ Dislocation & $101.00(24.10)$ & \\
\hline
\end{tabular}

Values are presented as mean (SD).

Rt.: right, Lt.: left, NSVD: normal spontaneous vaginal delivery. ${ }^{*} p<0.05$. 
Table 3. Correlation between treatment duration and variables

$(\mathrm{N}=63)$

\begin{tabular}{rcccccc}
\hline Variable & Age $(\mathrm{d})$ & A-SCMT $(\mathrm{mm})$ & N-SCMT $(\mathrm{mm})$ & A/N ratio & Head tilt $\left(^{\circ}\right)$ & Head rotation $\left({ }^{\circ}\right)$ \\
\hline Duration (d) & $-0.351^{*}$ & $0.351^{*}$ & 0.047 & 0.161 & $0.403^{* *}$ & -0.059 \\
\hline
\end{tabular}

Duration: treatment duration.

A-SCMT: affected side sternocleidomastoid muscle thickness, N-SCMT: non-affected side sternocleidomastoid muscle thickness, A/N ratio: affected side sternocleidomastoid muscle thickness/non-affected side sternocleidomastoid muscle thickness.

$*_{p}<0.01$.

Table 4. Factors affecting treatment duration

\begin{tabular}{crrrrr}
\hline Variable & $\mathrm{B}$ & $\beta$ & $\mathrm{t}$ & $p$-value & VIF \\
\hline Model 1 & & & & & \\
Constant & 40.078 & & 2.254 & 0.028 & 1.000 \\
Head tilt & 3.192 & 0.403 & 3.436 & 0.001 & \\
Model 2 & & & 3.355 & 0.001 & 1.036 \\
Constant & 69.562 & & 3.054 & 0.003 & 1.036 \\
Head tilt & 2.771 & 0.350 & -2.502 & 0.015 & \\
Age & -0.306 & -0.286 & & & \\
\hline
\end{tabular}

Model $1 \mathrm{R}=0.403, \mathrm{R}^{2}=0.162$, Adj $\mathrm{R}^{2}=0.148$.

Model $2 \mathrm{R}=0.491, \mathrm{R}^{2}=0.241$, Adj $\mathrm{R}^{2}=0.216$.

$1.81 \pm 0.28$, the SCM thickness on the non-affected side was $0.30 \pm 0.09$, the $\mathrm{A} / \mathrm{N}$ ratio of the $\mathrm{SCM}$ on the affected side was $6.33 \pm 1.53$, head tilt of $18.97 \pm 2.55$, and head rotation of $50.00 \pm 4.21$ (Table 1). There were no significant differences in treatment duration and sex, direction of the torticollis, delivery type, and hip dislocation ( $p>0.05$ ) (Table 2$)$. The duration of treatment was correlated with age, thickness of SCM on the affected side, and head tilt angle $(p<0.05)$ (Table 3$)$. The factors affecting the treatment duration were head tilt and age variables, and the modified $\mathrm{R}^{2}$ of Model 2 was 0.216 , which had $21 \%$ explanatory power (Table 4 ).

\section{Discussion}

There are reports that the incidence rate of torticollis is higher in males [3], and there are also reports that there is no difference between males and females $[18,19]$.

In this study, $46 \%$ of males and $53 \%$ of females were accounted for, but there was no significant difference between sex and treatment duration. According to researchers, there are studies showing that there are many SCM lesions are on the right side of the SCM [19] as well as the left side of the SCM $[20,21]$. In this study, the incidence was higher on the right with $62 \%$ compared to the left with $38 \%$, but there was no significant difference in the treatment duration.

The cause of torticollis is not clearly identified. However, according to the trauma theory, the cause of SCM damage caused by trauma during delivery was suggested [22]. However, there was no difference in the duration of treatment for torticollis according to the type of delivery between normal spontaneous vaginal delivery and cesarean section [15]. In one study, there was no significant difference between the two groups as a result of examining the significance of the treatment duration between the group with developmental dysplasia of the hip and the normal group [15].

In this study, there was no significant difference in treatment duration between the hip dislocation group and the normal group. The average age at which CMT is diagnosed is reported to be 2.3 months in one study [11] and 4 months in another study [21].

In this study, the date of diagnosis was 70.22 days and the average treatment duration was 100.62 days. This result was similar to the average treatment duration of the tumor group, and the treatment duration was longer than that of the muscular torticollis and postural torticollis [3].

In this study, the date of diagnosis was the same as the age of the patient, which was correlated with the treatment duration. It was found that there was a correlation between the diagnosis age of the patient and the treatment duration for torticollis [15], and the most important factor in the conservative treatment was the child's age [11].

As a result of the regression analysis of this study, age appeared as a factor in influencing the treatment duration, re- 
affirming the importance of treatment.

In other studies involving infants with an average diagnosis age of 36.63 days, SCM thickness and $\mathrm{A} / \mathrm{N}$ ratio did not show any correlation with rehabilitation duration [14]. In a study involving patients with an average diagnosis age of 68.80 days, the SCM thickness and $\mathrm{A} / \mathrm{N}$ ratio were correlated with the rehabilitation duration [15]. In this study of the mean diagnosis age was 70.22 days, the thickness of the affected SCM was found to be correlated with the treatment duration, but the $\mathrm{A} / \mathrm{N}$ ratio was not. The thickness of the non-affected SCM did not show any correlation with the treatment duration. The subjects of this study showed that the initial $\mathrm{A} / \mathrm{N}$ ratio was 3 times larger than that of Lee et al. [14] and Jung et al. [15], showing a large difference in thickness between the initial non-affected side and the affected side.

In order to minimize the child's posture and movement to one side, it is important to normalize the range of the cervical spine. A number of studies investigating torticollis have evaluated the functions of head tilt (or head bending) and head rotation with respect to neck movement $[10,15,23,24]$. In one study, there was no correlation between treatment duration and cervical rotation and side-bending [15]. In another study, the treatment duration and the angle of cervical rotation were divided into groups of more than 60 degrees and less than 60 degrees and 30 degrees and less than 30 degrees. As a result, the group with less rotation angle had the longest treatment duration and correlated with cervical range of motion (ROM) and treatment duration, which revealed that there is a relationship between cervical ROM and treatment duration [14].

In this study, the treatment duration and the head tilt angle were correlated, showing the same results. In one study, the smaller the difference between the affected side's rotational range and the normal side's rotational range, the greater the effectivness of treatement was shown, indicating that it is an important prognostic factor for torticollis [25]. However, in this study, only the rotation of the initial affected side was measured, and the same result was difficult to obtain because there was no difference from the non-affected side.

In this study, age, SCM thickness of affected side, head tilt and head rotation were the factors that showed correlation with the treatment duration of children under 3 months of age, but the main factors predicting the treatment duration were head tilt and age.

Restricted cervical rotation, measured by the ROM proportion of the affected side and the non-affected side, was a strong predictor and duration of treatment in the group without a mass, but was not significant in the group with a mass [20].

Although it was not measured with limited neck rotation in this study, the study subjects had a mass.

Babies at 1 month of age will develop the ability to incorporate reflexes and make their own movements to lift and hold the neck. In a study with an average diagnosis of 36.63 days, the treatment duration was significantly different depending on the degree of head rotation, the treatment duration and rotation had a correlation, and the initial average head rotation was 49.35 degrees [14]. In the study that included a CMT diagnosis average age of 68.80 days, there was no correlation between the treatment duration and head rotation, and the initial average head rotation was 73.46 degrees [15]. Therefore, in one study of subjects whose average diagnosis age was less than 3 months, the same results were not found on head rotation and treatment duration.

Head rotation is a measure of function, while head tilt can reflect the severity of CMT, showing the degree of asymmetry of the initial patient's neck tilt, and it has been found that there is a correlation between the treatment duration and the asymmetry [26].

Therefore, in order to shorten the treatment duration of children with CMT, early treatment should be initiated as various studies show the same results. Physical therapists needs to observe the changes head tilt angles of children with CMT. This has clinical significance that can be used as a tool to predict the treatment duration without undergoing expensive diagnosis or examination.

The study has a limitation of the inclusion of a small number of subjects who visited a local rehabilitation department to find out the characteristics of children under 3 months of age.

Future research will require studies to provide more detailed evidence with increased statistical power by including a larger sample size and classifying the characteristics according to the severity of CMT.

\section{Acknowledgements}

This paper was supported by Sahmyook University and was supported by the Center for Women In Science, Engineering and Technology (WISET) Grant funded by the Ministry of Science and ICT (MSIT) under the Program for Returners into R\&D. 


\section{Conflict of Interest}

The authors declared no potential conflicts of interest with respect to the authorship and /or publication of this article.

\section{References}

1. Rogers GF, Oh AK, Mulliken JB. The role of congenital muscular torticollis in the development of deformational plagiocephaly. Plast Reconstr Surg 2009;123:643-52.

2. Hollier L, Kim J, Grayson BH, McCarthy JG. Congenital muscular torticollis and the associated craniofacial changes. Plast Reconstr Surg 2000;105:827-35.

3. Cheng JC, Wong MW, Tang SP, Chen TM, Shum SL, Wong EM. Clinical determinants of the outcome of manual stretching in the treatment of congenital muscular torticollis in infants. A prospective study of eight hundred and twenty-one cases. J Bone Joint Surg Am 2001;83:679-87.

4. Davids JR, Wenger DR, Mubarak SJ. Congenital muscular torticollis: sequela of intrauterine or perinatal compartment syndrome. J Pediatr Orthop 1993;13:141-7.

5. Ho BC, Lee EH, Singh K. Epidemiology, presentation and management of congenital muscular torticollis. Singapore Med J 1999;40:675-9.

6. Cheng JC, Chen TM, Tang SP, Shum SL, Wong MW, Metreweli C. Snapping during manual stretching in congenital muscular torticollis. Clin Orthop Relat Res 2001;(384):237-44.

7. Staheli LT. Muscular torticollis: late results of operative treatment. Surgery 1971;69:469-73.

8. Cheng JC, Tang SP. Outcome of surgical treatment of congenital muscular torticollis. Clin Orthop Relat Res 1999;(362):190-200.

9. Rahlin M. TAMO therapy as a major component of physical therapy intervention for an infant with congenital muscular torticollis: a case report. Pediatr Phys Ther 2005;17:209-18.

10. Keklicek H, Uygur F. A randomized controlled study on the efficiency of soft tissue mobilization in babies with congenital muscular torticollis. J Back Musculoskelet Rehabil 2018;31:315-21.

11. Tatli B, Aydinli N, Caliskan M, Ozmen M, Bilir F, Acar G. Congenital muscular torticollis: evaluation and classification. Pediatr Neurol 2006;34:41-4.

12. Binder H, Eng GD, Gaiser JF, Koch B. Congenital muscular torticollis: results of conservative management with long-term follow-up in 85 cases. Arch Phys Med Rehabil 1987;68:222-5.

13. Demirbilek S, Atayurt HF. Congenital muscular torticollis and sternomastoid tumor: results of nonoperative treatment. J Pediatr Surg 1999;34:549-51.

14. Lee JY, Koh SE, Lee IS, Jung H, Lee J, Kang JI, et al. The cervical range of motion as a factor affecting outcome in patients with congenital muscular torticollis. Ann Rehabil Med 2013;37:18390 .

15. Jung AY, Kang EY, Lee SH, Nam DH, Cheon JH, Kim HJ. Factors that affect the rehabilitation duration in patients with congenital muscular torticollis. Ann Rehabil Med 2015;39:1824.

16. Lin $\mathrm{CH}$, Hsu HC, Hou YJ, Chen KH, Lai SH, Chang WM. Relationship between sonography of sternocleidomastoid muscle and cervical passive range of motion in infants with congenital muscular torticollis. Biomed J 2018;41:369-75.

17. Lee I. The effect of postural control intervention for congenital muscular torticollis: a randomized controlled trial. Clin Rehabil 2015;29:795-802.

18. Canale ST, Griffin DW, Hubbard CN. Congenital muscular torticollis. A long-term follow-up. J Bone Joint Surg Am 1982; 64:810-6.

19. Chang PY, Tan CK, Huang YF, Sheu JC, Wang NL, Yeh ML, et al. Torticollis: a long-term follow-up study. Zhonghua Min Guo Xiao Er Ke Yi Xue Hui Za Zhi 1996;37:173-7.

20. Emery C. The determinants of treatment duration for congenital muscular torticollis. Phys Ther 1994;74:921-9.

21. Wei JL, Schwartz KM, Weaver AL, Orvidas LJ. Pseudotumor of infancy and congenital muscular torticollis: 170 cases. Laryngoscope 2001;111(4 Pt 1):688-95.

22. Sanerkin NG, Edwards P. Birth injury to the sternomastoid muscle. J Bone Joint Surg Br 1966;48:441-7.

23. Ohman A, Mårdbrink EL, Stensby J, Beckung E. Evaluation of treatment strategies for muscle function in infants with congenital muscular torticollis. Physiother Theory Pract 2011;27: 463-70.

24. Li X, Li S, Pu B, Hua C. Comparison of 2 operative methods for treating laterocollis and torticollis subtypes of spasmodic torticollis: follow-up of 121 cases. World Neurosurg 2017;108:63641.

25. Cheng JC, Tang SP, Chen TM. Sternocleidomastoid pseudotumor and congenital muscular torticollis in infants: a prospective study of 510 cases. J Pediatr 1999;134:712-6.

26. Lee K, Chung E, Koh S, Lee BH. Outcomes of asymmetry in infants with congenital muscular torticollis. J Phys Ther Sci 2015; 27:461-4. 